\title{
General Clocks and the Clock Hypothesis
}

\author{
A. Sfarti \\ CS Dept, UC Berkeley, 387 Soda Hall, Berkeley \\ Email: egas@pacbell.net
}

\begin{abstract}
The present paper extends a previous proof of the Clock Hypothesis from the case of light clocks to clocks realized from oscillating massive particles. We also extend the study from the case of clocks undergoing constant proper acceleration to the case of clocks undergoing variable proper acceleration. We transformed the problem into one of general relativity and we applied the EulerLagrange formalism, thus providing a straightforward tool in solving this class of problems.
\end{abstract}

Keywords: Clock Hypothesis, general relativity, Schwarzschild metric, Euler-Lagrange formalism

PACS: 03.30.+p, 52.20.Dq, 52.70.Nc

\section{Introduction: the Lagrangian Method Applied to Radial Motion}

According to Rindler [1]:

"If an ideal clock moves non---uniformly through an inertial frame, we shall assume that acceleration as such has no effect on the rate of the clock, i.e. its instantaneous rate depends only on its instantaneous speed $v$ according to the above rule. This we call the clock hypothesis". (Rindler 1977, p43).

What Rindler refers to as "the above rule" is the standard time dilation formula that ties the proper time, the coordinate time and the speed:

$$
d \tau=d t \sqrt{1-\frac{v^{2}}{c^{2}}}
$$

Rindler allows that "certain natural clocks (vibrating atoms, decaying muons) conform very accurately to the clock hypothesis" [1] (page 43). There are both an experimental confirmation [2,3], [4-6] and a recent, very nice theoretical proof [7], for the case of light (electromagnetic) clocks under constant acceleration. In the current paper we set to extend Fletcher's proof to non-light clocks. i.e., clocks realized from massive particle oscillating between a pair of ideal mirrors while undergoing constant proper acceleration. For the case of uniform acceleration, we have transformed our problem from a special relativity problem of hyperbolic motion to a general relativity problem of clocks stationary in a uniform gravitational field by using the equivalence principle. The approach lends itself to some very easy to follow, elegant solutions based on the Euler-Lagrange variational methods. Throughout the paper we will consider that the acceleration vector coincides with the direction of motion and both are perpendicular on the two mirrors that form the clock. The mirrors are considered to be ideal, i.e. fully reflective.

The second part of our program is to study what happens in the case of non-uniform acceleration. In this case we can no longer appeal to the equivalence principle, since this principle applies exclusively to the equivalence between constant acceleration and (uniform) gravitational fields. Nevertheless, the Euler-Lagrange formalism, as applied to the Schwarzschild space developed in [8], produces valuable insight on whether the clock hypothesis applies to variable proper acceleration.

Throughout the second part of the paper we will use the Schwarzschild metric format for the particular case of absence of rotation $(d \theta=d \varphi=0)$ in order to describe the purely radial motion of the photons and of the massive particles making up the clocks. In all cases, the clock is made up of two mirrors moving independent of each other with the same proper acceleration thus maintaining a constant distance between them.

\section{Electromagnetic Clocks under Uniform Acceleration}


As explained in the introduction, we consider a light (electromagnetic) clock realized by two parallel mirrors undergoing the same proper acceleration and separated by the proper distance $d$. Instead of studying the complicated formalism describing the light clock kinematics in accelerated motion, we will use the equivalence principle in considering the mirrors stationary in a uniform gravitational field. A ray of light bouncing between the two mirrors follows the (null) geodesic of equation:

$$
\left(\frac{g r}{c^{2}}\right)^{2}(c d t)^{2}-d r^{2}=0
$$

In other words:

$$
d t=\frac{c}{g} \frac{d r}{r}
$$

We are interested in the proper time, not the coordinate time. The proper time for an observer attached to the mirrors stationary in the gravitational field (i.e. for $d r=0$ ) is:

In other words:

$$
(c d \tau)^{2}=\left(\frac{g r}{c^{2}}\right)^{2}(c d t)^{2}
$$

$$
d \tau=\frac{g r}{c^{2}} d t=\frac{d r}{c}
$$

So, we have proved that the oscillation period of the electromagnetic clock is independent of acceleration:

$$
T=2 \int_{r_{1}}^{r_{2}} d \tau=2 \frac{r_{2}-r_{1}}{c}=2 \frac{d}{c}
$$

\section{Generalization for Particle Clocks under Uniform Acceleration}

We now consider a clock realized by an arbitrary particle bouncing between two parallel mirrors undergoing the same proper acceleration and separated by the proper distance $d$. Towards the very end of his paper, Fletcher writes (without any proof): "In both the lemma and the theorem, it is clear that one can just as easily use timelike instead of null geodesics as the clock mechanism, i.e., bouncing massive particles instead of photons. But one must additionally require that the speed $v$ of the particles relative to the worldline whose proper time is to be measured be constant". In the following section we will demonstrate that speed does not need to be constant, it is a constant acceleration that satisfies the clock hypothesis. The trajectory of the bouncing particle is described by the geodesic that can be derived directly from the metric:

$$
\begin{aligned}
& d s^{2}=\alpha^{2} d t^{2}-d r^{2} \\
& \alpha=\frac{g r}{c}
\end{aligned}
$$

From the metric we obtain:

a) The Lagrangian [1]

$$
L=\alpha^{2} \frac{d t^{2}}{d s^{2}}-\frac{d r^{2}}{d s^{2}}
$$

As explained in [1], expression (3.2) is actually the square of the Lagrangian because it is easier to work with and it doesn't affect the expression of the Euler-Lagrange equations.

b) From the Lagrangian we obtain the Euler-Lagrange system of equations [1]:

$$
\begin{aligned}
& \frac{d}{d s}\left(\frac{\partial L}{\partial t}\right)-\frac{\partial L}{\partial t}=0 \\
& \frac{d}{d s}\left(\frac{\partial L}{\partial \dot{r}}\right)-\frac{\partial L}{\partial r}=0
\end{aligned}
$$

The over-dots signify derivative with respect to $s$. From (3.3) we obtain: 


$$
\begin{aligned}
& \alpha^{2} \frac{d t}{d s}=k \\
& -2 \frac{d^{2} r}{d s^{2}}-2 \frac{g^{2} r}{c^{2}}\left(\frac{d t}{d s}\right)^{2}=0
\end{aligned}
$$

In (3.4) $k$ is the total energy per unit of mass and the first equation signifies the conservation of total energy. The total angular momentum per unit mass [1] $h=r^{2} \frac{d \phi}{d s}$ is null in the case of radial motion and this falls out from the fact that the Lagrangian is independent of $\phi$ because the Schwarzschild metric is independent of $\phi$.

From the metric (3.1) we obtain:

$$
\begin{aligned}
& \alpha^{2}\left(\frac{d t}{d s}\right)^{2}=1+\left(\frac{d r}{d s}\right)^{2} \\
& d t=\frac{1+\left(\frac{d r}{d s}\right)^{2}}{k} d s
\end{aligned}
$$

The above is similar with the result shown in Landau and Lifshitz [9], eq (44.8) if we remember that the term $\alpha^{2} \frac{d t}{d s}=k$ represents the total (potential + kinetic) energy and $s$ is the general coordinate. The only difference is the absence of the square root, which is explained in [1] (see the explanation for the formula of the Lagrangian (3.2)).

Substituting (3.5) into (3.4) we obtain

c) The equation of motion:

$$
\frac{d^{2} r}{d s^{2}}=-\frac{1+\left(\frac{d r}{d s}\right)^{2}}{r}
$$

From (3.6) we can see that the acceleration increases as the radial coordinate decreases. In order to solve (3.6) we will need to resort to the fact that [8]:

Applying (3.7), equation (3.6) becomes:

$$
\frac{d^{2} r}{d s^{2}}=\frac{1}{2} \frac{d}{d r}\left(\frac{d s}{d r}\right)^{-2}
$$

$$
\frac{1}{2} \frac{d}{d r}\left(\frac{d s}{d r}\right)^{-2}=-\frac{\left(\frac{d r}{d s}\right)^{2}+1}{r}
$$

With the notation $y=\left(\frac{d s}{d r}\right)^{-2}$, equation (3.8) becomes:

with the immediate solution:

$$
\frac{1}{2} \frac{d y}{d r}=-\frac{\frac{1}{y}+1}{r}
$$

$$
y-\ln (1+y)=\ln \frac{r_{0}^{2}}{r^{2}}
$$

where $r_{0}=r(0)$ is the initial position of the particle oscillating between the mirrors.

Though we cannot find a symbolic solution for the above transcendental equation, we can easily see that the solution does not depend on the acceleration $g$. For $y<<1, \ln (1+y) \approx y-\frac{y^{2}}{2}$ so $(3.10)$ can be solved via approximation:

$$
y=\sqrt{\ln \frac{r_{0}^{2}}{r^{2}}}
$$


On the other hand, $y=\left(\frac{d r}{d s}\right)^{2}$, so (3.11) reduces to:

In other words:

$$
\frac{d s}{d r}=\frac{1}{\sqrt[4]{\ln \frac{r_{0}^{2}}{r^{2}}}}
$$

$$
c \frac{d \tau}{d r}=\frac{1}{\sqrt[4]{\ln \frac{r_{0}^{2}}{r^{2}}}}
$$

So, we get further confirmation that the clock period does not depend on the acceleration for the case of a clock made from a massive particle oscillating between the two mirrors moving with constant proper acceleration.

\section{Electromagnetic Clocks in a Non-Uniform Gravitational Field}

Though there is no correspondent equivalence principle for the case of non-uniform acceleration, it is interesting to study the case of non-uniform gravitational field in order to infer the behavior of clocks subjected to variable acceleration. For example, let's look at the case of the null geodesic in the Schwarzschild case (the null geodesic is the general relativity form of expressing the Maupertuis principle):

So:

$$
\begin{gathered}
\left(1-\frac{r_{s}}{r}\right)(c d t)^{2}-\frac{d r^{2}}{1-\frac{r_{s}}{r}}=0 \\
d t=\frac{1}{c} \frac{d r}{1-\frac{r}{r}}
\end{gathered}
$$

On the other hand, the proper time for an observer attached to the mirrors stationary in the gravitational field (i.e. for $d r=0$ ) is:

$$
d \tau=d t \sqrt{1-\frac{r}{r}}
$$

giving:

$$
d \tau=\frac{1}{c} \frac{d r}{\sqrt{1-\frac{r_{s}}{r}}}
$$

So, the oscillation period of the electromagnetic clock is:

$$
T=2 \int_{r_{1}}^{r_{2}} d \tau=\frac{2}{c}\left(r_{2} \sqrt{1-r_{s} / r_{1}}-r_{1} \sqrt{1-r_{s} / r_{2}}+r_{s} \ln \frac{2 r_{2}\left(1+\sqrt{1-r_{s} / r_{2}}\right)-r_{s}}{2 r_{1}\left(1+\sqrt{1-r_{s} / r_{1}}\right)-r_{s}}\right)
$$

If we consider $r_{1} \gg r_{s}, r_{2} \gg r_{s}$ and $r_{2}-r_{1}=d$ we obtain:

$$
T \approx \frac{2 d}{c}+\frac{r_{s}}{c} \ln \left(1+\frac{d}{r_{1}}\right) \approx \frac{2 d}{c}+\frac{r_{s}}{c} \frac{d^{2}}{r_{1}^{2}}
$$

We can conclude that there is a contribution, however small from the variable gravitational acceleration mediated by the Schwarzschild radius. On Earth, for a clock that has a $1 \mathrm{~m}$ distance between the mirrors, this contribution is negligible, of the order of less than $7.5^{*} 10^{-25}$ seconds. If we make the interval $r_{2}-r_{1}=d$ very small, then we can apply the equivalence principle and we can conclude, once again that

$$
T \approx \frac{2 d}{c}
$$


That is, the period of the clock is independent of its proper acceleration.

\section{Particle Clocks in a Non-Uniform Gravitational Field}

We start with the metric:

$$
\begin{aligned}
& d s^{2}=\alpha d t^{2}-\frac{1}{\alpha} d r^{2} \\
& \alpha=1-\frac{r}{r}
\end{aligned}
$$

According to reference [8], we obtain:

$$
\frac{d s}{d r}=\frac{1}{\sqrt{\frac{r_{s}}{r}-\frac{r_{s}}{r_{0}}}}
$$

where $r_{0}=r(0)$ is the initial position of the particle oscillating between the mirrors.

We can see that, again, there is a contribution from the gravitational acceleration mediated by the Schwarzschild radius.

$$
\tau=\frac{r_{0} \operatorname{arctg} \sqrt{\frac{r}{r_{0}-r}}-\sqrt{r\left(r_{0}-r\right)}}{c} \sqrt{\frac{r_{s}}{r_{0}}}
$$

So, once again, we see that the period of the clock is sensitive to the strength of the gravitational field. In a very small domain, we can apply the equivalence principle concluding that the period of the particle clock is independent of its proper acceleration.

\section{Relationship with the "Clock Ambiguity"}

During the review, a question has arisen: "What is the relationship, if any, between the Clock Hypothesis and the Clock Ambiguity?" The "Clock Ambiguity" [10] is a theory put forward by A. Albrecht and A. Islesias that relies on time independent Hamiltonians in order to postulate that there exists an "ambiguity" associated with the choice of clock in time reparameterization invariant theories. The answer is that there is no correlation between the two concepts, the "Clock Hypothesis" relies exclusively on time dependent Lagrangian / Hamiltonuian as derived from time dependent Schwarzschild solutions to Einstein field equations while the "Clock Ambiguity" relies on timeindependent Hamiltoniians. The thrust of our paper has been to reduce the Clock Hypothesis from the rank of postulate to the level of theorem.

\section{Conclusion}

We have reduced the hypothesis in the "Clock Hypothesis" to a provable theorem applicable not only to light (electromagnetic) clocks but also to clocks realized from massive particle oscillating between a pair of ideal mirrors while undergoing constant proper acceleration. We have derived some interesting conclusions for the case of light and massive particle clocks undergoing variable proper acceleration. By transforming the problem into one of general relativity, we have provided a simple, straightforward formalism for attacking the issue of proving the clock hypothesis in the case of generic (not light only) clocks.

\section{Acknowledgement}

The author is very grateful to the anonymous reviewer who challenged me to look into the equivalence of the Lagrangians/ Hamiltonians derived from the Schwarzschild metric and the ones presented by 
Landau and Lifshitz and for challenging me to examine the relationship between the Clock Hypothesis and the Clock Ambiguity.

\section{References}

1. W. Rindler, "Essential Relativity", (2nd ed.), New York, Springer---Verlag (1977).

2. J. Bailey et al., "Measurements of relativistic time dilation for positive and negative muons in a circular orbit," Nature, 268 (July 28, 1977).

3. J. Bailey et al., "Final report on the CERN muon storage ring including the anomalous magnetic moment and the electric dipole moment of the muon, and a direct test of relativistic time dilation," Nuclear Physics B,150 (1979).

4. S.R. Mainwaring, G.E. Stedman, "Accelerated Clock Principles in Special Relativity," Phys. Rev., A47 (1993).

5. R. Anderson, I. Vetharaniam, G.E. Stedman, "Conventionality of Synchronization, Gauge Dependence and Test Theories of Relativity," Physics Reports, 295 (1998).

6. A.M. Eisele, "On the Behavior of an Accelerated Clock", Helvetica Physica Acta 60 (1987).

7. S. C. Fletcher, "Light Clocks and the Clock Hypothesis," Found Phys, 43, (2013).

8. A. Sfarti, "Euler-Lagrange Solution for Calculating Particle Orbits in Gravitational Fields," Fizika A, 19, 4, (2010).

9. L .D. Landau, E. M. Lifshitz, "Mechanics - Course of Theoretical Physics," vol 1, 3th ed, Elsevier edit., (2007).

10. A. Albrecht, A. Islesias, "The Clock Ambiguity: Implications and New Developments," The Fundamental Theories of Physics, 172, Springer Verlag, (2012). 